\title{
Integrated transcriptome and proteome analysis reveals complex regulatory mechanism of cotton in response to salt stress
}

CHEN Lin ${ }^{1+}$, SUN Heng ${ }^{1,2+}$, KONG Jie ${ }^{3}$, XU Haijiang ${ }^{3 *}$ and YANG Xiyan ${ }^{1 *}$

\begin{abstract}
Background: Soil salt stress seriously restricts the yield and quality of cotton worldwide. To investigate the molecular mechanism of cotton response to salt stress, a main cultivated variety Gossypium hirsutum L. acc. Xinluzhong 54 was used to perform transcriptome and proteome integrated analysis.

Results: Through transcriptome analysis in cotton leaves under salt stress for $0 \mathrm{~h}$ (T0), $3 \mathrm{~h}$ (T3) and $12 \mathrm{~h}$ (T12), we identified 8 436, 11628 and 6311 differentially expressed genes (DEGs) in T3 vs. T0, T12 vs. T0 and T12 vs. T3, respectively. A total of 459 differentially expressed proteins (DEPs) were identified by proteomic analysis, of which 273, 99 and 260 DEPs were identified in T3 vs. T0, T12 vs. T0 and T12 vs. T3, respectively. Metabolic pathways, biosynthesis of secondary metabolites, photosynthesis and plant hormone signal transduction were enriched among the identified DEGs or DEPs. Detail analysis of the DEGs or DEPs revealed that complex signaling pathways, such as abscisic acid (ABA) and jasmonic acid (JA) signaling, calcium signaling, mitogen-activated protein kinase (MAPK) signaling cascade, transcription factors, activation of antioxidant and ion transporters, were participated in regulating salt response in cotton.
\end{abstract}

Conclusions: Our research not only contributed to understand the mechanism of cotton response to salt stress, but also identified nine candidate genes, which might be useful for molecular breeding to improve salt-tolerance in cotton.

Keywords: Cotton, Proteome, Salt stress, Transcriptome

\section{Background}

Soil salinity caused by high concentration of salt ions in soil, which seriously restricts the agricultural production. More than 800 million hectares of land and $20 \%$ of the arable land throughout the world are affected by salt stress, which will continue

\footnotetext{
*Correspondence: xhj863@163.com; yxy@mail.hzau.edu.cn

${ }^{+}$Chen $\mathrm{L}$ and Sun $\mathrm{H}$ contributed equally to this work.

${ }^{3}$ Institute of Economic Crops, Xinjiang Academy of Agricultural Sciences, Urumqi 830091, Xinjiang, China

${ }^{1}$ National Key Laboratory of Crop Genetic Improvement, National Center of Plant Gene Research (Wuhan), College of Plant Science and Technology,

Huazhong Agricultural University, Wuhan 430070, Hubei, China

Full list of author information is available at the end of the article
}

to deteriorate (Al Murad et al. 2020; Munns and Tester 2008). It was showed that the problem of soil salinity become more and more serious in China. The area of saline-alkali land in China is about 100 million hectares. Moreover, the area of secondary salinization in the western region and the Yellow River Delta are increased 150 000 200 000 and $100000 \mathrm{hm}^{2}$ every year, respectively. The high concentration of salt ions can change soil structure, reduce soil permeability and water conductivity, trigger different levels of stress response on plants, such as osmotic stress, ion cytotoxicity, nutritional deficiency, and oxidative stress (Mahajan and Tuteja 2005; Muchate et al.

(c) The Author(s). 2021 Open Access This article is licensed under a Creative Commons Attribution 4.0 International License, which permits use, sharing, adaptation, distribution and reproduction in any medium or format, as long as you give appropriate credit to the original author(s) and the source, provide a link to the Creative Commons licence, and indicate if changes were made. The images or other third party material in this article are included in the article's Creative Commons licence, unless indicated otherwise in a credit line to the material. If material is not included in the article's Creative Commons licence and your intended use is not permitted by statutory regulation or exceeds the permitted use, you will need to obtain permission directly from the copyright holder. To view a copy of this licence, visit http://creativecommons.org/licenses/by/4.0/ 
2016; Zelm et al. 2020). Osmotic stress reduces water uptake and leads to water deficit in plants, which occurs in the early stage of salt stress. Ion cytotoxicity is caused by the high concentration of salt ions $\left(\mathrm{Na}^{+}\right)$in the cytoplasm, and it disrupts the uptake of other ions and obstructs some metabolic pathways, such as photosynthesis (Wang et al. 2020; Zhang et al. 2019). Osmotic stress and ionic stress can cause secondary stress in plants, for example, the high concentration of reactive oxygen species will cause damage to cell structures and biological macromolecules (Yang and Guo 2018a).

The salt tolerance of plants depends on their ability to extract water and nutrients from saline soils and to avoid excessive accumulation of salt ions in plant tissues (Zorb et al. 2019). Most of crop plants are salt-sensitive, and the yield of crops will be seriously reduced when they are subjected to moderate salt stress (Park et al. 2016; Zorb et al. 2019). Therefore, analyzing the mechanism of plant response to salt stress and cultivating new salt-tolerant crop varieties are very important to ensure the sustainability of agricultural production and food security.

Cotton (Gossypium) is the most important fiber crop, and China is a larger producer and the largest consumer of cotton in the world. Xinjiang is the main cotton-producing area in China, and more than $32.6 \%$ of cultivated land in Xinjiang is affected by different degrees of salinization stress, which seriously threatens the safety of cotton production. It is very important to improve salt tolerance based on ensuring high yield and quality in cotton. Transcriptome and proteome have made progress in revealing the mechanism of salt tolerance and identifying candidate genes in cotton (Gong et al. 2017; Guo et al. 2015; Li et al. 2015; Peng et al. 2014; Shan et al. 2019; Sikder et al. 2020; Yang et al. 2019). As there are multi-level regulatory machineries exist in saltstress response, including transcription and translation regulations, it is important to monitor the gene expression level of RNA and protein simultaneously. Fortunately, the development of integrated transcriptome and proteome makes this research strategy possible (Chen et al. 2015; Peng et al. 2018; Trevisan et al. 2015; Wang et al. 2014; Yang et al. 2019).

In the present study, an integrated transcriptome and proteome analysis were performed to deeply investigate the molecular mechanism of cotton in response to salt stress. There were 15822 DEGs and 459 DEPs identified in mRNA transcription and protein expression level, respectively, and a total of 164 stress-response associated DEGs and DEPs were identified. And important biological pathways related to salt-stress response were elucidated. Furthermore,
9 candidate genes were identified by integrating proteomic and transcriptomic profiles. Our results will further enrich the understanding of molecular mechanism in cotton in response to salt stress.

\section{Materials and methods}

\section{Plant materials and treatments}

The widely planted upland cotton variety Xinluzhong 54 (Gossypium hirsutum L. acc.) was used in the research, and it was provided by Xinjiang Academy of Agricultural Sciences (Additional file 1: Figure S1). Cotton seedlings were cultured in Hoagland solution under $16 \mathrm{~h}$ light $/ 8 \mathrm{~h}$ dark conditions. For salt stress treatment, $250 \mathrm{mmol} \cdot \mathrm{L}^{-1} \mathrm{NaCl}$ was added to the solution at two-leaf stage seedlings to simulate salt stress. The second newly developed leaves were collected at $0 \mathrm{~h}$ (T0), $3 \mathrm{~h}$ (T3) and $12 \mathrm{~h}$ (T12) for subsequent RNA and protein isolations.

\section{RNA sequencing (RNA-seq) and isobaric tags for relative and absolute quantification (iTRAQ) analysis}

Total RNAs were extracted using the RNAprep Pure Plant Kit (Cat. \#DP441, TIANGEN). RNA-Seq was conducted using Illumina Hiseq platform (BGI Tech, Shenzhen, China). After removing the low-quality, adaptor-polluted and high content of unknown base (N) reads, the filtered reads were mapped against Gossypium hirsutum (acc. TM-1) genome and genes were annotated accordingly (Zhang et al. 2015). The gene expression levels for each sample were calculated with RSEM software, the DEGs were detected with DEseq2 (Fold change $\geq 2$, adjusted $P$-value $\leq 0.05)$.

For iTRAQ analysis, the protein quantitative analysis is carried out by BGI company (BGI Tech, Shenzhen, China), and the main processes includes: protein extraction and digestion, peptide labeling and fractionation, protein quantification by LC-MS / MS. The quantitative analysis was performed by IQuant software (Wen et al. 2014). T3 vs. T0 (T3/ T0), T12 vs. T0 (T12/T0) and T12 vs. T3 (T12/T3) were set as comparison groups, the DEPs were identified using the threthold of fold change $>1.2$ (or $<0.83$ ) and Q-value $<0.05$. Both RNA-Seq and iTRAQ experiments were performed with three biological replicates.

\section{Functional enrichment analysis}

KEGG (Kyoto encyclopedia of genes and genomes) and GO (Gene ontology) functional enrichment analysis of DEGs and DEPs were performed using the "Gene-list enrichment" program in KOBAS3.0 database (http://kobas.cbi.pku.edu.cn/anno_iden.php) (Xie et al. 2011). Transcription factors were identified by 
PlantTFDB 4.0 (http://planttfdb.cbi.pku.edu.cn/). The protein interaction networks were predicted by STRI NG software (ver. 11.0), and were visualized by Cytoscape software (ver. 3.4.0). The gene/protein expression patterns were showed by heatmap with the expression values normalized by Genesis software.

\section{Quantitative real-time PCR (qRT-PCR) analysis}

High-quality cDNA was obtained by reverse transcription using SuperScript III Reverse Transcriptase following the manufacturer's instructions (Cat. No.18080-093, Invitrogen). Gene expression levels were detected using an ABI Prism 7500 system (Applied Biosystems). GhUBQ7 (GenBank accession No. DQ116441) was used as the internal control. Gene-specific primers for qRT-PCR were designed according to the cDNA sequences using Primer Premier 5.0 software and synthesized commercially (Genscript Bioscience) (Additional file 1: Table S1).

\section{Results}

Identification of DEGs and DEPs during cotton response to salt stress

The seedlings of Xinluzhong 54 (Gossypium hirsutum L. acc.) were cultured in Hoagland solution and 250 $\mathrm{mmol} \cdot \mathrm{L}^{-1} \mathrm{NaCl}$ was added to simulate salt stress. At the two-leaf stage, the second newly-developed leaves were used for transcriptome and proteome analysis, the workflow of experimental design and analysis was shown in Fig. 1a. Three samples with three biological replicates were analyzed using Illumina Hiseq platform and iTRAQ system.

The average output data of each sample from RNA-Seq was 6.64 Gb. A Phred quality score (Q score) was used to evaluate the base call accuracy of the read data. Q20 represents an incorrect base call of 1 in 100 (99\%), Q30 represents an incorrect base call of 1 in 1000 (99.9\%) and Q30 is usually used as an evaluation standard in the quality control of sequencing data. Here, the average Q20 and Q30 of each sample were 97.77 and $93.65 \%$, respectively. The clean reads were mapped to the reference genome of Gossypium hirsutum (TM-1), and the average alignment rate of each sample is $82.83 \%$ (Additional file 1: Table S2). DEseq2 algorithm was used to detect the DEGs with the standard of fold change $\geq 2$ and $P$-value $\leq 0.05$. There were 8436 (4 123 upregulated, 4313 down-regulated), 11628 (4 649 upregulated, 6979 down-regulated) and 6311 (2 169 up-regulated, 4142 down-regulated) DEGs were identified in $\mathrm{T} 3 / \mathrm{T} 0, \mathrm{~T} 12 / \mathrm{T} 0$ and $\mathrm{T} 12 / \mathrm{T} 3$, respectively (Fig. 1b). DEGs were distributed more in D

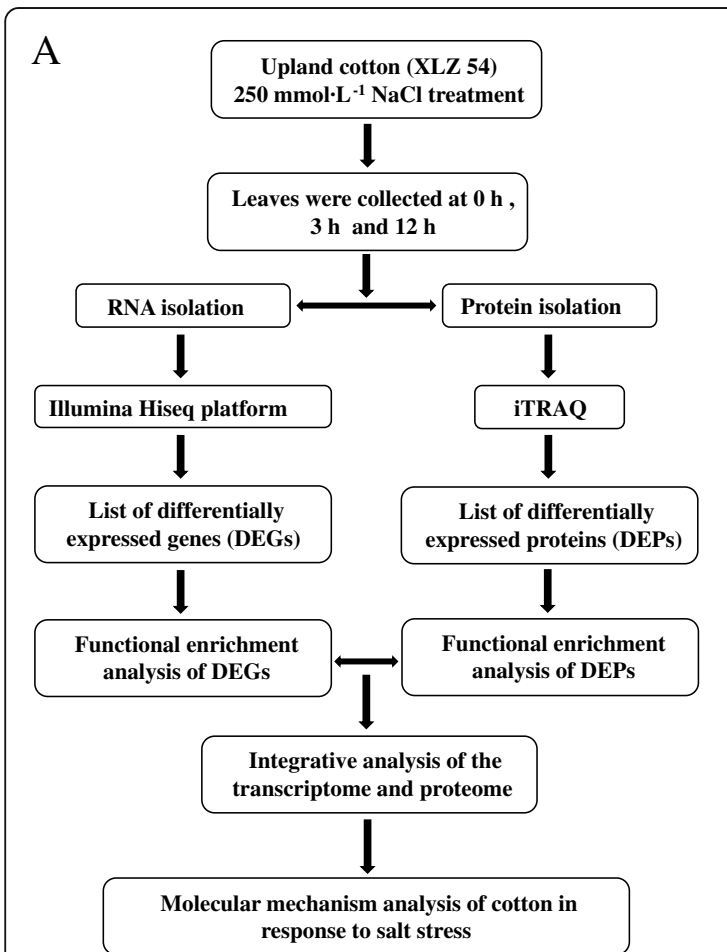

B

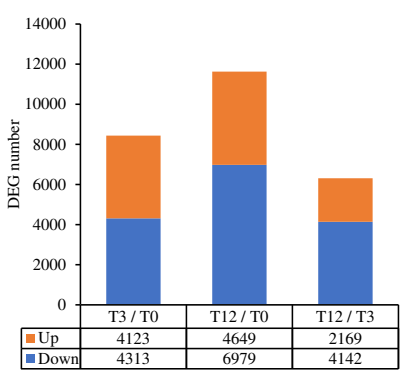

$\mathrm{C}$

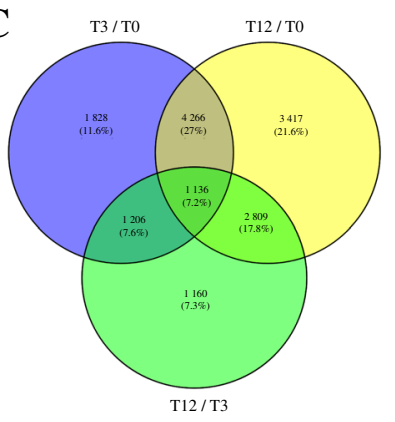

D

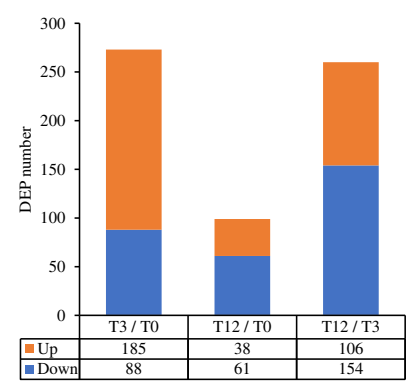

$\mathrm{E}$

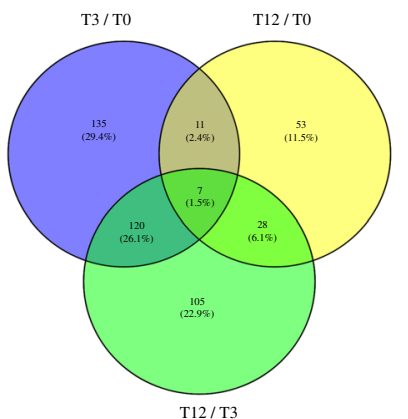

Fig. 1 Identification of differentially expressed genes (DEGs) and proteins (DEPs) in response to salt stress. a Scheme of experimental design in integrated transcriptome and proteome analysis. b Number of DEGs in different comparison groups. c Venn diagram of DEGs. $\mathbf{d}$ Number of DEPs in different comparison groups. e Venn diagram of DEPs 
sub-genome (53\%) than A sub-genome. And more DEGs were distributed on chromosomes A05 and D05, while less were distributed on A04 and D03 (Additional file 1: Figure S2a). Twenty-five genes were randomly selected to evaluate the RNA-Seq results by $\mathrm{qRT}-\mathrm{PCR}$, there was a significant correlation between these two groups of data $\left(R^{2}=0.874\right)$ (Additional file 1: Figure S2b). It indicated that the sequencing data of the RNA-Seq had high reliability, and could be used for subsequent analysis. Venn diagram showed that 5402,3945 and 2342 common DEGs were detected in T3 / T0 and T12 / T0, T12 / T0 and T12 / T3, T3 / T0 and T12 / T3, respectively, with 1136 common DEGs shared by the three comparisons (Fig. 1c). The 1136 common DEGs showed up-regulated and down-regulated expression patterns by calculating hierarchical clustering analysis, with up-regulated genes enriching in galactose metabolism, biosynthesis of secondary metabolites, metabolic pathways, valine, leucine and isoleucine degradation, glucosinolate biosynthesis, and downregulated genes in metabolic pathways, carbon fixation in photosynthetic organisms, biosynthesis of secondary metabolites, carbon metabolism and flavonoid biosynthesis (Additional file 1: Figure S3a).

A total of 459 DEPs were identified by iTRAQ, and 273 (185 up-regulated, 88 down-regulated), 99 (38 upregulated, 61 down-regulated) and 260 (106 upregulated, 154 down-regulated) DEPs were identified in
T3/T0, T12/T0 and T12/T3, respectively (Fig. 1d). All DEPs encoding genes were equally distributed on A subgenome and D sub-genome, and more DEPs were distributed on chromosomes A05 and D05. Venn diagram showed that 18, 127 and 35 common DEPs in T3/T0 and T12/T0, T3/T0 and T12/T3, T12/T0 and T12/T3, respectively (Fig. 1e). There were seven common DEPs in three groups, three proteins (Gh_A02G0551, Gh A07G0867, Gh_D08G1902) were up-regulated at $3 \mathrm{~h}$, two proteins (Gh_D02G2126, Gh_A03G1705) were down-regulated at $3 \mathrm{~h}$ and up-regulated at $12 \mathrm{~h}$, and $\mathrm{Gh}_{-}$ Sca010764G01 was up-regulated at $3 \mathrm{~h}$ and $12 \mathrm{~h}, \mathrm{Gh}$ D11G1672 was down-regulated at $3 \mathrm{~h}$ and $12 \mathrm{~h}$.

\section{Integration of proteomic and transcriptomic profiles}

To investigate the multi-level regulation of gene expression under salt stress, an integrative analysis of the transcriptome and proteome was performed in this study. Results showed that 77, 70 and 47 associated DEGs and DEPs were identified in T3/T0, T12/T0 and T12/T3, respectively (Fig. 2a, Additional file 1: Table S3). In T3/T0, 31 and 46 genes showed the same and opposite expression patterns at the transcriptional level and translational level, respectively. In T12/T0, 62 and 8 genes showed the same and opposite expression patterns at these two levels, respectively. In T12/T3, 31 and 16 genes showed the same and opposite expression patterns at these two levels, respectively (Fig. 2a). These results suggested that genes in response to salt stress may

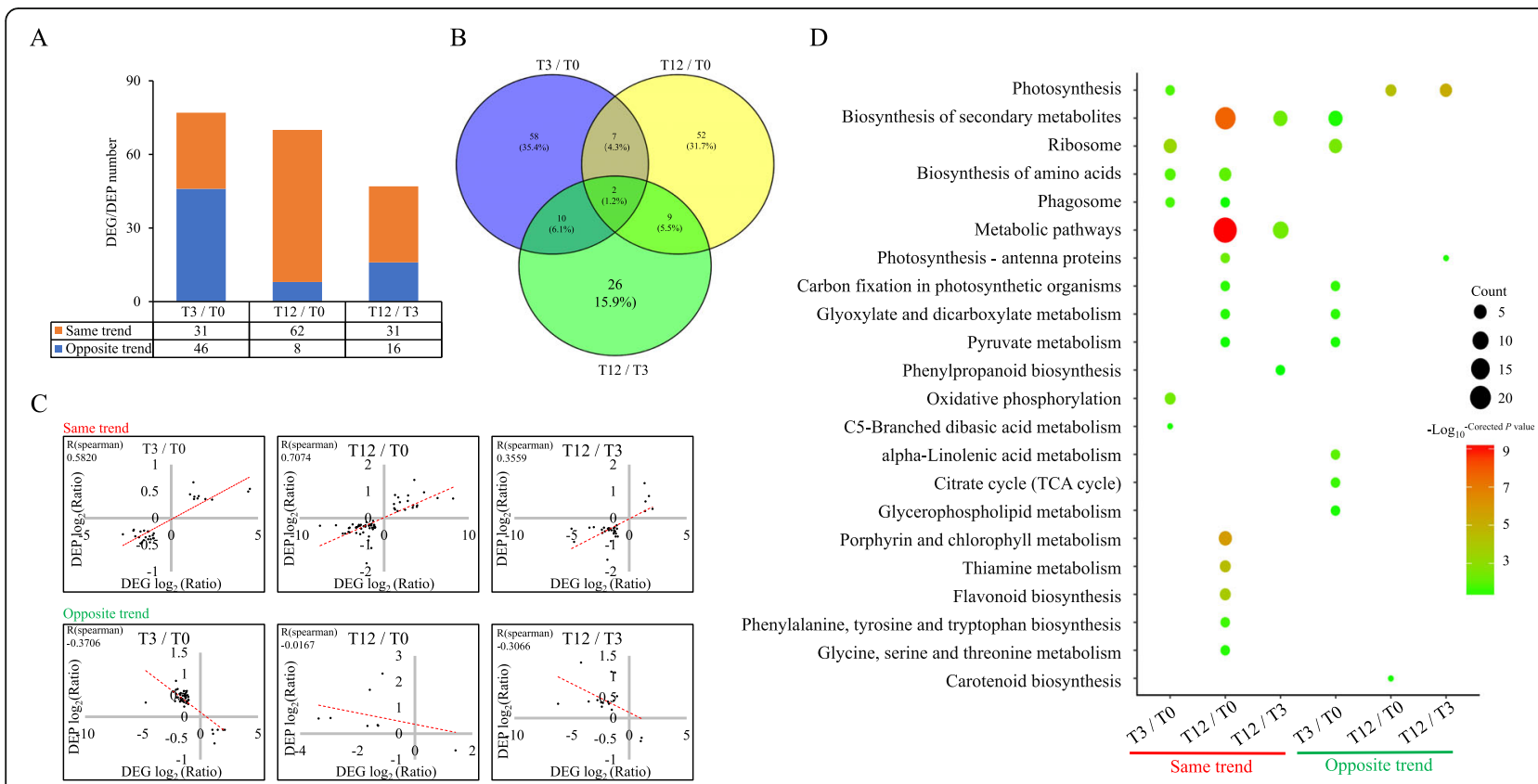

Fig. 2 Statistics and functional analysis on the correlation between DEGs and DEPs. a Statistics on the number of correlated DEGs and DEPs. b Venn diagram of correlated DEGs/DEPs. c Concordance between the gene changes at transcriptome and proteome levels. $\mathbf{d}$ Functional enrichment analysis of the correlated DEGs and DEPS 
undergo complex regulation at the transcriptional and translational levels.

Venn diagram showed that nine common, associated genes (Gh_D08G1902, Gh_A07G0867, Gh_Sca010764G01, Gh_D13G1729, Gh_D09G1072, Gh_D06G2351, Gh_ A11G0835, Gh_D02G2126, Gh_A03G1705) were found in the comparion group of T3/T0 and T12/T0. Eleven associated genes (Gh_D11G1672, Gh_D10G2299, Gh_D06G1799, Gh_D05G2157, Gh_A11G1830, Gh_A10G1991, Gh_A06G1466, Gh_A05G1452, Gh_A01G1839, Gh_D02G2126, Gh_A03G1705) were identified in T12/T0 and T12/T3. And twelve associated genes (Gh_D09G1409, Gh_D06G0164, Gh_D04G0628, Gh_D02G0438, Gh_A04G0778, Gh_A03G1240, Gh_A01G1605, Gh_D05G0122, Gh_A05G1494, Gh_A03G0173, Gh_D02G2126, Gh_A03G1705) were identified in T3/T0 and T12/T3 (Fig. 2b). All associated genes were listed in Additional file 1: Table S4. Spearman correlation analysis showed that a poor correlation between the expression changes of these associated DEGs and DEPs was observed $(R:-0.37 \sim-0.71)$ (Fig. 2c).

\section{Functional enrichment analysis of DEGs and DEPs}

KEGG analysis was performed to study the functional enrichment of up-regulated and down-regulated DEGs in each comparison group. For up-regulated DEGs, 21, 30 and 4 pathways were enriched in T3/T0, T12/T0 and T12/T3, respectively (Additional file 1: Figure S3b). Plant hormone signal transduction, biosynthesis of secondary metabolites and metabolic pathways occurred simultaneously in these three groups. For down-regulated DEGs, 17, 22 and 25 pathways were enriched in T3/T0, T12/T0 and T12/T3, respectively (Additional file 1: Figure S3c). Ribosome was the most significantly enriched pathway in T3/T0, and metabolic pathways was significantly enriched in T12/T0 and T12/T3.

For up-regulated DEPs, 8,2 and 14 pathways were enriched in $\mathrm{T} 3 / \mathrm{T} 0, \mathrm{~T} 12 / \mathrm{T} 0$ and $\mathrm{T} 12 / \mathrm{T} 3$, respectively (Corrected $P \leq 0.01$ ) (Additional file 1: Figure S4a). Photosynthesis and pyruvate metabolism were the common pathways in these three groups. Glyoxylate and dicarboxylate metabolism, ribosome, carbon fixation in photosynthetic organisms and citrate cycle (TCA cycle) were significantly enriched in T3/T0. In addition, ten pathways were enriched in T12/T3, such as endocytosis, alpha-linolenic acid metabolism and glycerophospholipid metabolism. For the downregulated DEPs, 11, 13 and 9 pathways were enriched in $\mathrm{T} 3 / \mathrm{T} 0, \mathrm{~T} 12 / \mathrm{T} 0$ and $\mathrm{T} 12 / \mathrm{T} 3$, respectively (Additional file 1: Figure S4b). Metabolic pathways and biosynthesis of secondary metabolites were the common pathways in these three groups. In addition, selenocompound metabolism, biosynthesis of amino acids and porphyrin and chlorophyll metabolism were commonly enriched in T3/T0 and T12/T0, photosynthesis and ribosome were commonly enriched in T3/T0 and T12/T3, flavonoid biosynthesis and carbon fixation in photosynthetic organisms were commonly enriched in T12/T0 and T12/T3.

KEGG analysis showed that these associated genes/ proteins were enriched in multiple pathways (Corrected $P$-value $\leq 0.05$ ) (Fig. 2d). We found some DEGs showed the same changing patterns at transcriptional and protein levels and were enriched in some pathways, such as flavonoid biosynthesis (Gh_D08G1902, Gh_A10G1079, Gh_A05G1424, Gh_A12G0549), porphyrin and chlorophyll metabolism (Gh_A09G1143, Gh_A05G3766, Gh_ D01G1828, Gh_A10G0282, Gh_A10G2274), thiamine metabolism (Gh_A06G1269, Gh_A05G1432, Gh_D05G1605), biosynthesis of amino acid (Gh_D08G2317, Gh_A13G1464, Gh_D13G1473, Gh_D06G1578, Gh_D01G1099, Gh D05G2157, Gh_D09G0576).

\section{Disturbance of energy metabolism caused by salt stress}

The carbohydrate metabolism pathways and photosynthesis were very important for the storage and utilization of energy in plants. Here, the changes of DEGs and DEPs which were involved in these two biological processes were analyzed. Treated with salt stress, cotton genes involved in the glycolysis/ gluconeogenesis pathways exhibited strong expression levels. The expression levels of GhPFK3 (Gh A05G0198), GhPKP2 (Gh_A08G0871) and GhPKP1 (Gh_A10G1036) were up-regulated under salt stress. GhHXK3 (Gh_D06G0003), a gene that encodes hexokinase (HK), was down-regulated under salt stress, with the transcription levels decreased to 22 and $9.6 \%$ of control at $3 \mathrm{~h}$ and $12 \mathrm{~h}$, respectively (Fig. 3a). The protein abundance of enolase PGH1 (Gh Sca286293G01), fructose-bisphosphate aldolase FBA5 (Gh_D13G1361), pyruvate dehydrogenase subunit (Gh_A12G1299) and triosephosphate isomerase (Gh_ D01G1600) increased significantly at $3 \mathrm{~h}$ after salt stress (Additional file 1: Figure S5a). The acetaldehyde dehydrogenase protein (Gh_D06G1578) increased significantly at $12 \mathrm{~h}$, hexokinase HK (Gh_A13G1742) and ethanol dehydrogenase (Gh_A01G1605) protein decreased at $3 \mathrm{~h}$.

The down-regulation was observed in DEGs encoding isocitrate dehydrogenase (IDH) (GhCICDH:, Gh_A11G1562), pyruvate dehydrogenase E1 subunit (GhMAB1:, Gh_A12G1299) and malate dehydrogenase (GhMMDH2: Gh_A01G04040, GhMMDH1: Gh_A04G0320) which were involved in the tricarboxylic acid (TCA) cycle (Fig. 3b). And the abundance of malate dehydrogenase (Gh_D02G0438, Gh_ A02G0386, Gh_D05G3328) and pyruvate dehydrogenase E1 subunit (Gh_A12G1299) increased at $3 \mathrm{~h}$ after salt stress (Additional file 1: Figure S5b). The protein abundance of succinate dehydrogenase 
A
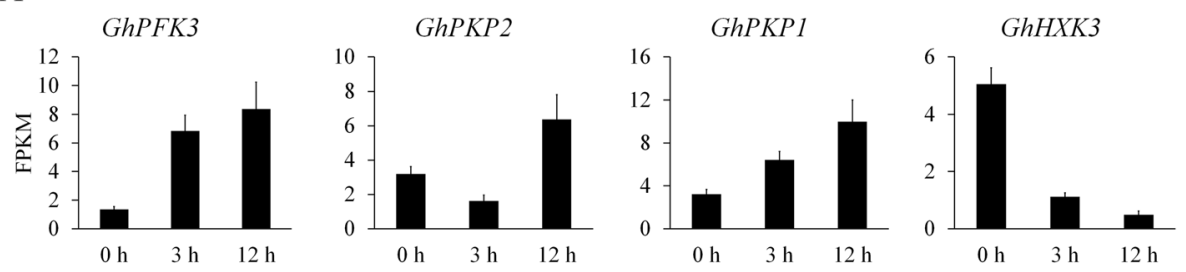

B
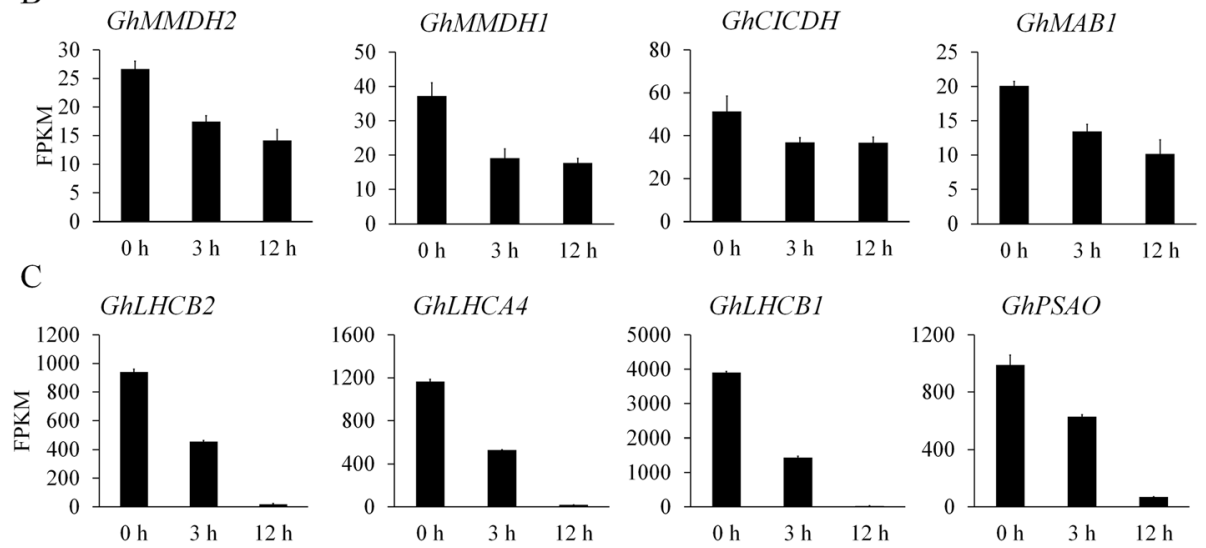

Fig. 3 The expression patterns of functional genes which were involved in energy metabolism. a The expression patterns of genes involved in the glycolysis/gluconeogenesis pathways. b The expression patterns of genes involved in tricarboxylic acid cycle. $\mathbf{c}$ The expression patterns of genes involved in photosynthesis

subunit (Gh_A10G1110) and 2-oxoglutarate dehydrogenase (Gh_A05G3057) decreased at $3 \mathrm{~h}$ (Additional file 1: Figure S5b).

Similarly, the expression levels of genes modulating photosynthesis was significantly downregulated in cotton. As photosystem I subunit PSAO related gene, GhPSAO (Gh_D07G1090) and light-harvesting chlorophyll protein complex LHC-related genes, GhLHCB1 (Gh_D06G2351), GhLHCB2 (Gh_A07G1725) and GhLHCA4 (Gh A12G1617) (Fig. 3c). The protein abundances of most DEPs involved in photosynthesis were increased under salt stress (Additional file 1: Figure S5c). For example, photosystem II subunit PSBO (Gh_D11G1897), PSBP (Gh_ A05G3293, Gh_D04G0311) and PSBQ2 (Gh_A10G2195) proteins increased at $3 \mathrm{~h}$ under salt stress. The abundance of photosystem I related proteins psaA (Gh_A01G1364, Gh_A09G0964) and psaB (Gh_A12G1136, Gh Sca005646G01) decreased after $3 \mathrm{~h}$ of salt stress, but increased at $12 \mathrm{~h}$. Taken together, these results suggested that salt stress can cause the changes in expression level of cotton genes involved in carbohydrate metabolism pathways and photosynthesis pathways, to enhance the tolerance and survival of cotton in salt stress.

\section{Signal transduction in response to salt stress in cotton} Plant hormone signal transduction

Plant hormones play an important role in the regulation of plant salt stress resistance. Here, the expression changes of key genes in different hormone signaling pathways were analyzed (Figure S6). ABA and JA hormone signals are important for plants to resist salt stress. Most of the genes involved in ABA signaling pathway, such as PP2C, SnRK2 and $\mathrm{ABF}$, were up-regulated by salt stress (Fig. 4a). We speculated that the continuous activation of ABA signal may play an important role in cotton response to salt stress. ABF transcription factors play an important role in regulating $A B A$ signal transduction. Fourteen genes encoding $\mathrm{ABF}$ transcription factors were identified, all of them were up-regulated by salt stress (Fig. 4a). Nine ABF genes (Gh_D05G2495, Gh_A05G2234, Gh_D12G0214, Gh_A05G1751, Gh_D05G1946, Gh_D02G0909, Gh_ A03G1664, Gh_A03G2095, Gh_D02G2079) were continuously induced by salt stress during $0-12 \mathrm{~h}$, and the maximum expression levels of four genes (Gh_A12G0212, Gh_D13G0400, Gh_A13G0355, Gh_A13G2006) were detected at $3 \mathrm{~h}$ (Fig. 4a).

The JAR1 gene in JA pathway encodes a jasmonic acid-amino synthase, which could catalyze the formation of bioactive jasmonic acid-isoleucine (JA-Ile) in Arabidopsis. The JAR1 gene in cotton, Gh_A08G1120 and Gh_D08G1403, were significantly up-regulated in T3/ T0, so we speculated that salt stress might induce JA-Ile synthesis and activate JA signal in the early stage (Fig. 4b). MYC2 is an important transcription factor in JA signaling pathway in Arabidopsis. We identified two genes encoding MYC2 transcription factors that were 


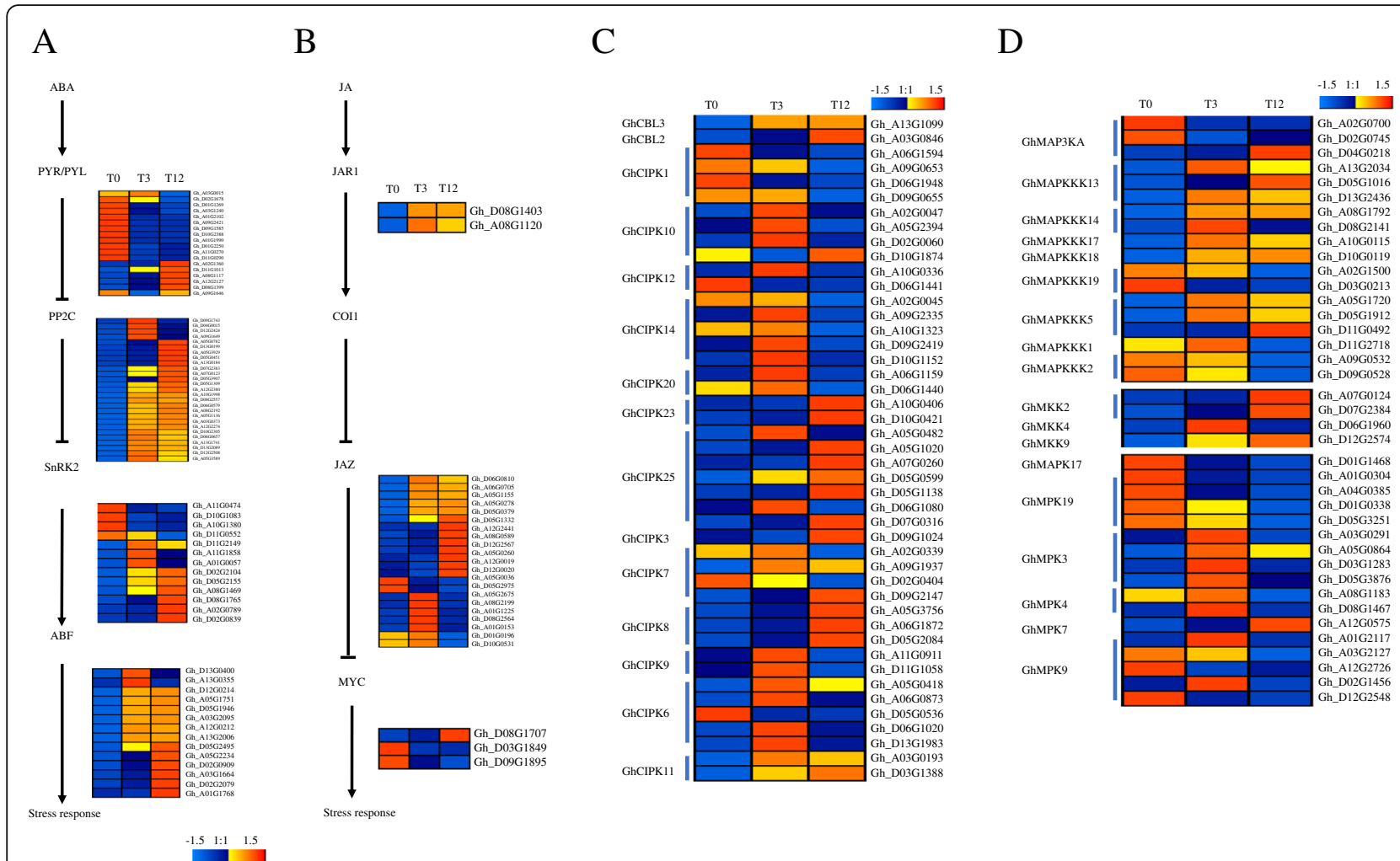

Fig. 4 Plant signal transduction in response to salt stress in cotton. $\mathbf{a}-\mathbf{b}$ The expression patterns of genes which were involved in ABA and JA signal pathways. $\mathbf{c}$ The expression patterns of CBL and CIPK genes. $\mathbf{d}$ The expression patterns of MAPK signaling cascade genes

differentially expressed, Gh_D08G1707 was significantly induced by salt stress and showed continuous expression patterns, while Gh_D09G1895 was down-regulated by salt stress. Furthermore, 21 differentially expressed JAZ genes were identified, among them, 18 genes were upregulated (Fig. 4b).

\section{Transcription factor}

Transcription factor plays an important role in plant response to abiotic stress. There were 696 (up-regulated: 429, down-regulated: 267), 926 (up-regulated: 492, down-regulated: 434) and 532 (up-regulated: 232, down-regulated: 300) transcription factors identified in T3/T0, T12/T0 and T12/T3, respectively (Figure $\mathrm{S} 7 \mathrm{a}$ and $\mathrm{b})$. Among these transcription factors, NAC, ERF, MYB, bZIP and WRKY family genes accounted for a large proportion. Among the transcription factors with the spatio-temporal expression patterns, about 32 and $49 \%$ were only differentially expressed at $3 \mathrm{~h}$ and $12 \mathrm{~h}$ after salt stress, respectively (Additional file 1: Figure S7a and b). Some of the transcription factors that were revealed to be responsive to salt stress previous studies were also detected in this study (Gao et al. 2020; Krishnamurthy et al. 2020; Xie et al. 2010; Zhao et al. 2019) (Additional file 1: Figure S7c).

\section{Calcineurin B-like (CBL)-interacting protein kinase (CIPK) signaling cascade}

The CBL-CIPK signaling system can participate in the process of plant stress response by sensing calcium ion to regulate intracellular signal transduction. Two genes encoded CBL, GhCBL2 (Gh_A03G0846) and GhCBL3 (Gh_A13G1099), were identified, both were significantly up-regulated by salt stress. There were 43 CIPK genes identified, and most of these genes were up-regulated by salt stress (Fig. 4c). We predicted the potential protein interaction models between these CBL and CIPK proteins through the STRING database. Results showed that these two CBL proteins could interact with multiple CIPK proteins, respectively (Additional file 1: Figure S8a). Furthermore, some CBL and CIPK genes were found with similar expression patterns under salt stress, such as CBL2 and CIPK8 (Gh_A05G3756, Gh_A06G1872, Gh_D05G2084), both types were continuously induced by salt stress.

\section{Mitogen-activated protein kinase signaling cascade}

Mitogen-activated protein kinase (MAPK) cascade signaling pathway is composed of three kinds of serine/threonine protein kinases, MAP3K, MKK and MPK. There were 18, 4 and 17 genes encoded MAP3K, MKK, MPK identified, respectively (Fig. 4d). Most of the MAPK pathway genes were up-regulated by salt stress at $3 \mathrm{~h}$. 
The potential protein interaction models were also predicted through the STRING database. Two up-regulated genes, Gh_A07G0124 and Gh_D07G2384, which encoding GhMKK2, were predicted wide interaction models with upstream MAP3K proteins and downstream MPK proteins. We speculated that GhMKK2 might play an important role in regulating cotton response to salt stress through MAPK cascade pathway (Additional file 1: Figure S8b).

\section{Antioxidant and ion transport systems}

Salt stress causes the imbalance of reactive oxygen species (ROS) homeostasis and activate the ROS scavenging mechanism. NADPH oxidase is involved in the production of ROS in plants under stress. Four genes (GhRBOHA; Gh_A02G1791, Gh_A12G2669, Gh_ D03G0688 and Gh_D12G2750) encoding NADPH oxidase respiratory burst oxidase homolog A (RBOHA) were up-regulated by salt stress (Fig. 5a). Consistently, dramatic up-regulation was detected in genes encoding catalase (GhCAT2), superoxide dismutase (GhFSD2) and glutathione S-transferase (GhGSTT1, GhGSTZ1) (Fig. 5b). Our results indicated that pathways involved of CAT, SOD and GST genes might play an important role in protecting cotton from oxidative damage caused by salt stress.

The regulation of intracellular ion homeostasis is very important for plants response to salt stress. Ion channel

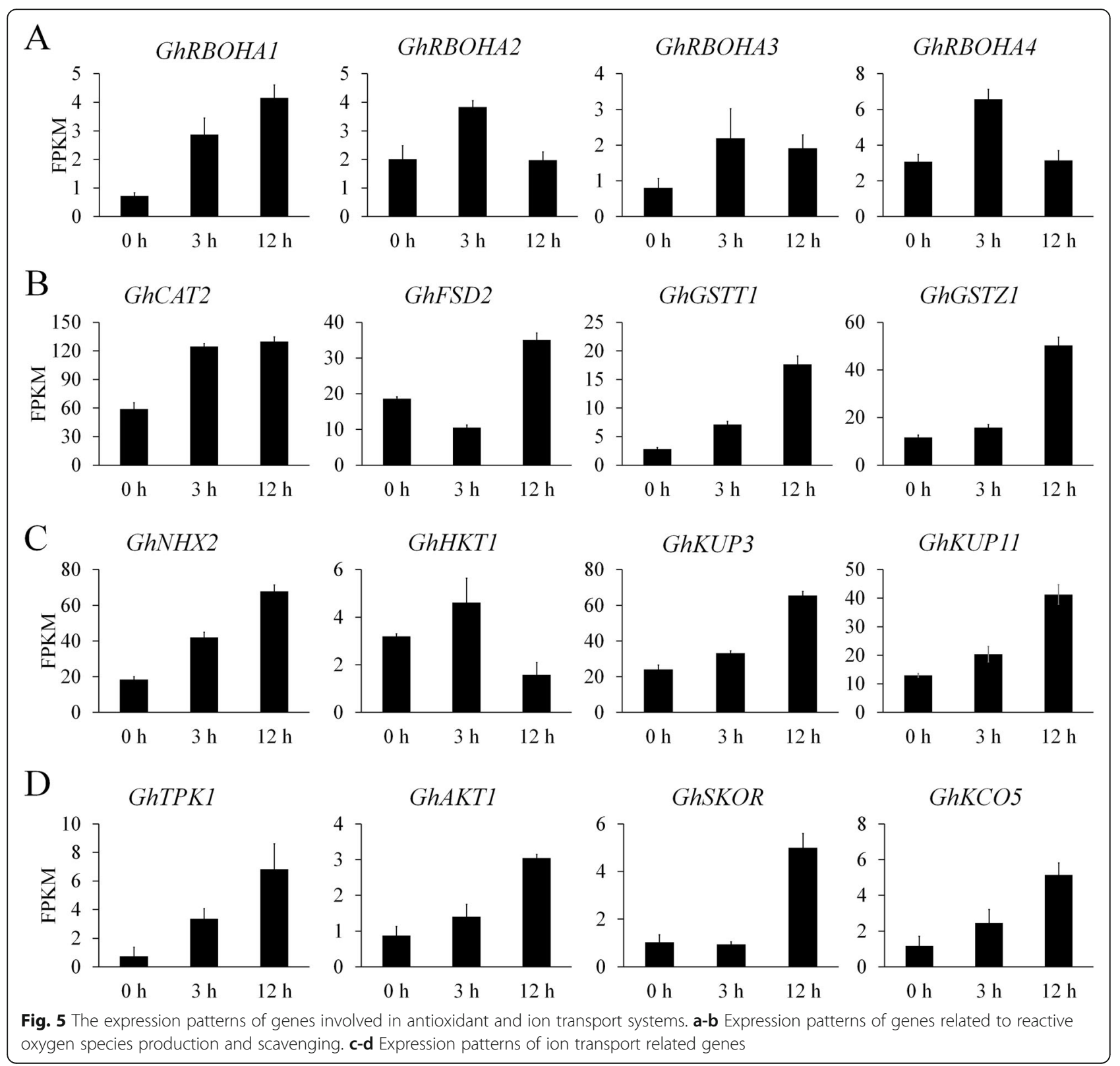


protein $\left(\mathrm{Na}^{+} / \mathrm{H}^{+} / \mathrm{K}^{+}\right.$transporter) are tightly linked to plant response to salt stress. Thus, we analyzed the expression of genes that encoding ion transporters. GhNHX2 (Gh_A11G2132) and GhKUP3 (Gh D04G0700), GhKUP11 (Gh_A12G0438) encoding $\mathrm{Na}^{+} /$ $\mathrm{H}^{+}$transporters and $\mathrm{K}^{+}$transporters, were significantly up-regulated under salt stress (Fig. 5c). GhHKT1 (Gh A08G2473) encoding $\mathrm{Na}^{+}$transporters were downregulated under salt stress at $12 \mathrm{~h}$. Potassium channels are involved in the regulation of ion absorption and transport. And significant up-regulation at $12 \mathrm{~h}$ under salt stress were observed in genes that encoding inward rectifying potassium channel (GhAKT1, Gh_D13G2111) and outward rectifying $\mathrm{K}^{+}$channel (GhTPK1, Gh D01G0726; GhKCO5, Gh_A11G0947; GhSKOR, Gh_ D05G1041) (Fig. 5d).

\section{The identification of candidate genes by integrating proteomic and transcriptomic profiles}

Based on the gene expression and functional annotation, we identified 9 candidate genes that might play an important role in cotton response to salt stress, i.e., GhLEA14 (Gh_A11G0835), GhKCS6 (Gh_A03G1286), GhPRXR1 (Gh_A05G1452), GhPOD (Gh_A08G0714), GhFIB (Gh_A05G1494), GhALDH7B4 (Gh_D06G1578), GhUSP (Gh_A03G0386) and GhAVP1 (Gh_D05G0122), GhADH1 (Gh_A01G1605) (Table 1). GhLEA14 encodes late embryogenesis abundant protein and its expression level was up-regulated at both transcriptional and protein levels under salt stress. This gene might be involved in the regulation of osmotic stress and the prevention of oxidant stress in cotton undersalt stress. The peroxidase superfamily protein GhPRXR1 and GhPOD were upregulated at transcription and protein levels by salt stress, two correspondent genes might be involved in the hydrogen peroxide catabolic process. GhADH1 and
GhALDH7B4 encoding ethanol dehydrogenase and acetaldehyde dehydrogenase, respectively. These two genes were up-regulated at the transcriptional and protein levels under salt stress. We speculated that these two genes may be involved in the cellular detoxification of cotton under salt stress.

\section{Discussion}

Complex regulatory mechanisms took place when plants encountered salt stress. Although cotton is an inherently salt-tolerant crop, with the aggravation of soil salinization in its planting area, it is important to analyze the molecular mechanism of its response to salt stress and to cultivate novel cotton varieties with higher salt tolerance. Due to the high yield, perfect fiber quality and disease resistance, Xinluzhong 54 is one of the main cotton variety grown in Xinjiang nowadays (Additional file 1: Figure S1a). However, soil salinity is one of the major factors restricting the further expansion of Xinluzhong 54 (Additional file 1: Figure S1b and c). Firstly, it is necessary to clarify the molecular mechanism of Xinluzhong 54 in response to salt stress. Here, an integrated analysis of transcriptome and proteome was performed to analyze the regulation mechanism of cotton in response to salt stress and identified the key regulatory genes which may be used to improve the salt tolerance in cotton.

Plants initiate defense response against salt stress by regulating the expression of stress responsive genes. It has been widely reported that phytohormones were involved in regulating plant response to salt stress (Ryu and Cho 2015). Transcriptome analysis revealed that cotton response to salt stress induced a variety of hormone signaling pathway genes, reflecting the complexity of hormone involved in the regulation of cotton response to salt stress (Additional file 1: Figure S6). Some

Table 1 Candidate genes involved in salt stress response in cotton

\begin{tabular}{|c|c|c|c|c|c|}
\hline Name & Gene_ID & Comparison group & DEG $\log _{2}$ (FoldChange) & DEP $\log _{2}$ (FoldChange) & Description \\
\hline \multirow[t]{2}{*}{ GhLEA14 } & Gh_A11G0835 & T3/T0 & 4.53 & 0.55 & Late embryogenesis abundant protein \\
\hline & & $\mathrm{T} 12 / \mathrm{TO}$ & 2.84 & 0.85 & \\
\hline GhKCS6 & Gh_A03G1286 & T12/T0 & 3.57 & 1.43 & 3-ketoacyl-CoA synthase 6 \\
\hline \multirow[t]{2}{*}{ GhPRXR1 } & Gh_A05G1452 & $\mathrm{T} 12 / \mathrm{T3}$ & 1.72 & 0.82 & Peroxidase superfamily protein \\
\hline & & T12/T0 & 1.68 & 0.77 & \\
\hline GhPOD & Gh_A08G0714 & $\mathrm{T} 3 / \mathrm{TO}$ & 1.58 & 0.41 & Peroxidase superfamily protein \\
\hline \multirow[t]{2}{*}{ GhFIB } & Gh_A05G1494 & $\mathrm{T} 3 / \mathrm{TO}$ & 1.28 & 0.67 & Fibrillin precursor protein \\
\hline & & $\mathrm{T} 12 / \mathrm{T3}$ & -2.38 & -0.43 & \\
\hline GhALDH7B4 & Gh_D06G1578 & T12/T0 & 2.48 & 0.31 & Aldehyde dehydrogenase \\
\hline GhUSP & Gh_A03G0386 & T12/T0 & 1.20 & 0.37 & Encodes universal stress protein \\
\hline GhAVP1 & Gh_D05G0122 & $\mathrm{T} 12 / \mathrm{T} 3$ & 1.42 & 0.62 & Inorganic H pyrophosphatase family protein \\
\hline GhADH1 & Gh_A01G1605 & $\mathrm{T} 12 / \mathrm{T} 3$ & 2.03 & 0.34 & Alcohol dehydrogenase \\
\hline
\end{tabular}

Note: The $\log _{2}$ (FoldChange) value indicates the relative change in abundance of candidate genes's transcripts at different time point is shown as a log ${ }_{2}$ ratio 
evidences suggested that $\mathrm{ABA}$ and JA hormone can enhance salt tolerance in many plants (Goossens et al. 2016; Vishwakarma et al. 2017). ABA signaling pathway was activated by salt stress, it was consistent with the increase of ABA content in cotton leaves ( $\mathrm{Li}$ et al. 2019). In addition, some reports have pointed out that JA also plays an important role in regulating salt tolerance in other plants, such as rice and wheat (Kang et al. 2005; Qiu et al. 2014). A previous study showed that genes involved in JA synthesis and signal transduction were induced by salt stress, such as $L O X, A O S, J A Z$ and $M Y C$. Appropriate concentrations $\left(0.01 \mu \mathrm{mol} \cdot \mathrm{L}^{-1}\right)$ of coronatine, which is structurally and functionally similar to JA, could improve the salt tolerance of cotton (Xie et al. 2015). Overexpression of the JA signal repressor gene GhJAZ2 in cotton significantly reduces the salt tolerance (Sun et al. 2017). The cross regulation between ABA and JA plays an important role in mediating salt tolerance. PnJAZ1, a moss jasmonate ZIM-domain gene is induced by $A B A$, enhances salt tolerance in plants through inhibiting ABA signaling under salt stress (Liu et al. 2019). The cytokinin regulatory pathway is different from $A B A$ and JA, it has been shown that genes in cytokinin signaling pathways are down-regulated, which are consistent with a previous report (Liu et al. 2012). We speculated that the inhibition of cytokinin signaling may be one of the reasons for repressing cotton growth under salt stress.

Some genes involved in signal transduction were differentially activated or repressed under salt stress, such as transcription factors, CBL interaction protein kinases, MAPK signaling cascade pathway related genes (Fig. 4c and d, Additional file 1: Figure S7). Many transcription factor family genes showed rapid response to salt stress. It was remarkable that most of the differentially expressed NAC genes were up-regulated when cotton encountered salt stress, it is consistent with the previous studies that NAC genes were widely involved in salt response (Sun et al. 2018). Overexpression of salt stress induced NAC gene GHATAF1 enhances the salt tolerance of cotton (He et al. 2016). Transcriptome analysis showed that CBL interaction protein kinase and MAPK signaling genes were induced by salt stress (Fig. 4c, d). In Arabidopsis, CBL2/3 either regulated osmotic or salt stress responses by interacting with CIPK21 and targeting the kinase to the vacuole thus regulating the homeostasis of ions and water on the vacuole (Pandey et al. 2015), or regulated potassium and magnesium ion homeostasis by interacted with CIPK3, CIPK9 and CIPK23 (Liu et al. 2013; Tang et al. 2015). Here, two CBL genes (GhCBL2, GhCBL3), which were homologous to Arabidopsis $C B L 2$ and $C B L 3$, were up-regulated by salt stress and were predicted to interact with several salt-induced CIPK proteins, such as CIPK3, CIPK9 and
CIPK23 (Figure S8a). In Arabidopsis, the interaction between MKK2 and MPK4 enhanced salt stress tolerance through MEKK1-MKK2-MPK6/MPK4 pathway (Teige et al. 2004). MAPK cascade MEKK1-MKK2-MPK6/ MPK4 could cross-regulate plant response to salt stress with phytohormones, it is involved in SA- and ROSinitiated stress signaling, and plays a vital role in maintaining ROS homeostasis under salt stress (Yang and Guo 2018b). In cotton, the expression patterns of MAPK cascade genes were significantly induced after salt stress treatment at $4 \mathrm{~h}$ (Chen et al. 2020). In this study, the homologous genes of Arabidopsis MKK2 and MPK4 genes were up-regulated by salt stress (Fig. 4d). We speculated that some conservative regulatory mechanisms may exist between cotton and Arabidopsis.

Salt stress induced the accumulation of reactive oxygen species and caused lipid peroxidation. In this study, some antioxidant enzyme genes were up-regulated under salt stress (Fig. 5b), which were consistent with the activation of some antioxidant enzymes in previous studies (Meloni et al. 2003). Salt stress induced the increase of $\mathrm{Na}^{+}$content in cotton leaves (Peng et al. 2014). Here, we identified some transporters and channel proteins encoding genes that showed differently expressed under salt stress, they might play an important role in regulating $\mathrm{Na}^{+}$homeostasis under salt stress (Fig. 5c). Salt stress induced stomatal closure, photosynthetic rate declined, energy metabolism inhibited in plants. Consistent with previous studies, many DEGs and DEPs involved in these biological processes and showed down-regulated expression patterns under salt stress.

Our results showed that only 77, 70 and 47 differentially expressed genes were associated with mRNA and protein levels in T3/T0, T12/T0 and $\mathrm{T} 12 / \mathrm{T} 3$, respectively (Fig. 2a). Previous studies have shown that there was a low correlation between transcriptome and proteome results (Chen et al. 2017; Wang et al. 2015). For example, transcriptional factors, CBL-CIPK and MAPK cascade related genes which were identified in transcriptome, were not identified in proteome. The nonlinear and low correlation between these two omics may be due to the following reasons: (i) Post-transcriptional and post-translational modification; (ii) The different rates between transcription and translation, and the different half-life between mRNA and proteins; (iii) The threshold used in the identification of DEGs and DEPs.

However, an integrated analysis of transcriptome and proteome could give more information both on mRNA and protein levels. In the present study, the DEGs which were simultaneously identified at the transcriptional and protein levels were selected for identifying candidate genes. Based on this research strategy, 9 candidate genes that might play an important role in cotton 


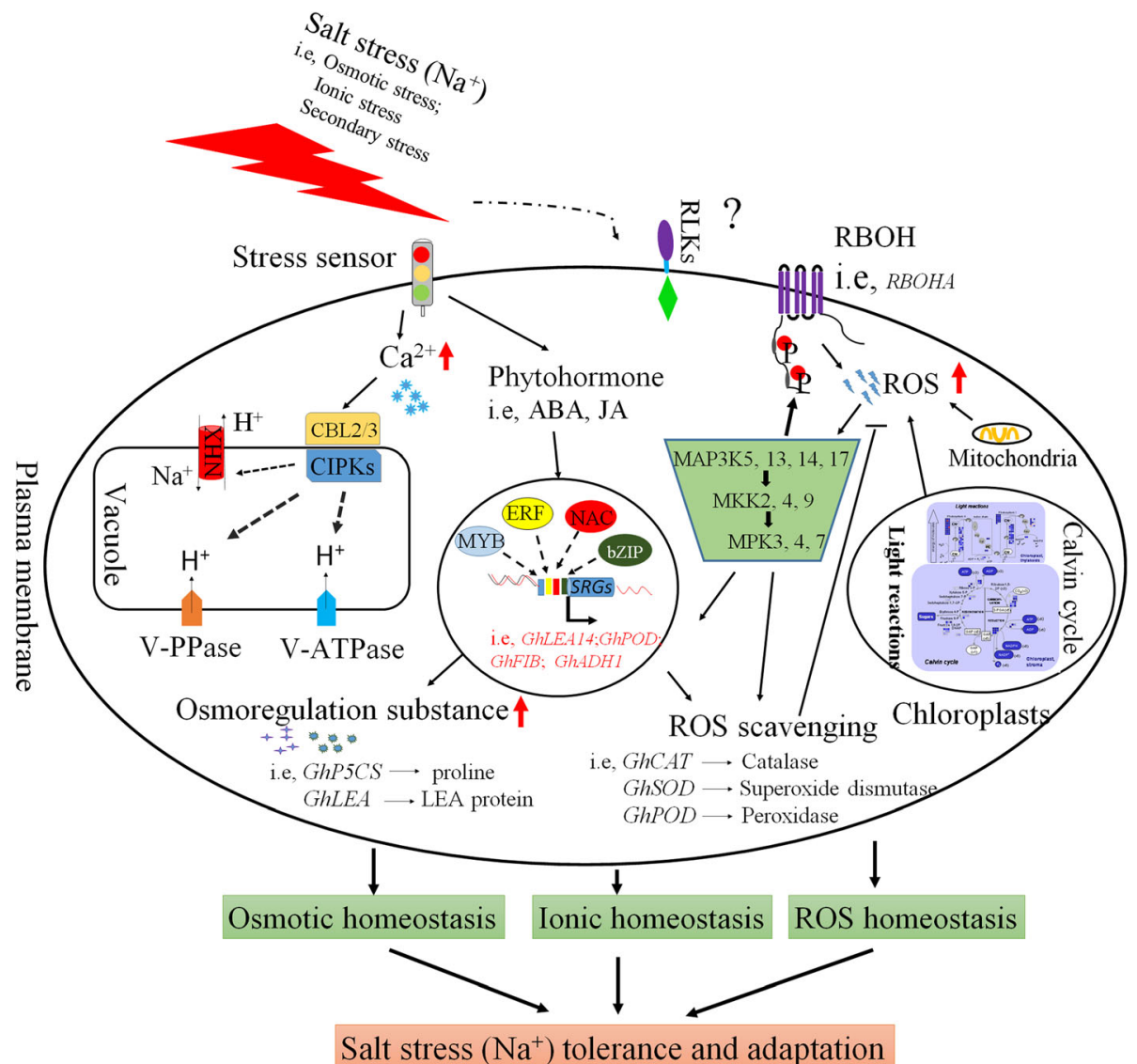

Fig. 6 Schematic presentation of a possible regulatory model in cotton (Gossypium hirsutum L. acc. Xinluzhong 54) leaves under salt stress. Cells of cotton sense salt stress by RLKs or other unknown sensors, then transfer the external signal to the cytoplasm, and change the content of $\mathrm{Ca}^{2+}$, ROS and other hormones (i.e, ABA, JA). Inside of the cell, $\mathrm{Ca}^{2+}$ signal cascade (i.e, GhCBL2,3; GhCIPK8,9,10,25), ROS, MAPK cascade (i.e, GhMAP3K5,13,14,17-GhMKK2,4,9-GhMPK3,4,7) and other hormone signal cascades pathway are activated, which could alter the global transcriptional profiles in cotton (the expression of stress related transcription factors, such as MYB, ERF, NAC and bZIP are initiated). The expression of genes encoding proteins that related to salt stress response, including NHX, osmoregulation related and ROS scavenging proteins (i.e, GhLEA14; GhP5CS; GhPOD; GhSOD; GhCAT; GhFIB; GhADH1). Ultimately, pathways to maintain the osmotic homeostasis, ionic homeostasis and ROS homeostasis are activated to facilitate cotton to resist and adapt to salt stress

response to salt stress were identified (Fig. 6). AtLEA14 (AT1G01470) is the homologous gene of GhLEA14 (Gh_A11G0835) in Arabidopsis, and the amino acid sequence homology between GhLEA14 and AtLEA14 is $70.86 \%$. Overexpression of AtLEA14 could significantly enhance the salt stress tolerance of Arabidopsis (Jia et al. 2014). We speculate that GhLEA14 is very likely to regulate salt tolerance in cotton. These candidate genes can provide important resources for genetic improvement of salt tolerance in cotton.

\section{Conclusions}

According to the integrated analysis of transcriptome and proteome, a complex salt stress response network was outlined in cotton. This study can contribute to understand the mechanism of cotton response to salt stress and facilitate the breeding of salt-resistant cotton varieties by molecular breeding.

\section{Supplementary Information}

The online version contains supplementary material available at https://doi. org/10.1186/s42397-021-00085-5.

\section{Additional file 1: Figure S1. Description of Xinluzhong 54. a} Cultivation area of Xinluzhong 54. b-c Plant phenotype of Xinluzhong 54 under $150 \mathrm{mmol} \cdot \mathrm{L}^{-1}$ and $250 \mathrm{mmol} \cdot \mathrm{L}^{-1} \mathrm{NaCl}$ treatment, respectively. Figure S2. Identification of differentially expressed genes (DEGs) in response to salt stress. $\mathbf{a}$ The number of DEGs on each chromosome. $\mathbf{b}$ qRT-PCR validation of transcript levels evaluated by RNA-Seq. Figure $\mathbf{S 3}$. Functional enrichment analysis of DEGs. a Expression patterns and enrichment pathways of 1136 common DEGs. b KEGG analysis of up-regulated DEGs. c KEGG analysis of down-regulated DEGs. Figure S4. Functional enrichment analysis of DEGs. a KEGG analysis of up-regulated DEPs. $\mathbf{b}$ KEGG analysis of down-regulated DEPs. Figure S5. Expression patterns of differentially expressed proteins involved in photosynthesis and carbon catabolism. a-c stand for the expression patterns of DEPs involved in 
photosynthesis, glycolysis/ gluconeogenesis and TCA cycle, respectively. Figure S6. Plant hormone signal pathways analysis. The expression changes of key node genes involved in different hormone signal pathway under salt stress treatment. Figure S7. Transcription factors response to salt stress in cotton. $\mathbf{a}$ and $\mathbf{b}$ stand for the statistics of up-regulated and down-regulated transcription factors induced by salt stress, respectively. c The expression patterns of different transcription factors under salt stress treatment. Figure S8. Potential protein interaction models through the STRING database. a Predicted CBL-CIPK protein interaction patterns. b Predicted MAPK signal cascade protein interaction patterns. Table S1. Primers used for qRT-PCR. Table S2. Transcriptome data statistics. Table S3. Statistics on the correlation between DEGs and DEPs. Table S4. Information of associated genes.

\section{Acknowledgments}

We would like to thank the anonymous reviewers for their valuable comments and helpful suggestions, which have helped to improve the manuscript.

\section{Authors' contributions}

Chen $L$ and Sun $H$ performed the experiments, analyzed the data and wrote the manuscript. Kong J provided the seeds and performed the salt stress assay in the field. Yang XY and $\mathrm{Xu} \mathrm{HJ}$ conceived this project and revised the manuscript. All authors read and approved the final manuscript.

\section{Funding}

This work was supported by National R\&D Project of Transgenic Crops of Ministry of Science and Technology of China (2016ZX08005-004-002).

\section{Availability of data and materials}

The datasets used in this study are available from the corresponding author on reasonable request.

\section{Declarations}

Ethics approval and consent to participate

Not applicable.

\section{Consent for publication}

Not applicable.

\section{Competing interests}

The authors declare that they have no competing interests.

\section{Author details}

'National Key Laboratory of Crop Genetic Improvement, National Center of Plant Gene Research (Wuhan), College of Plant Science and Technology, Huazhong Agricultural University, Wuhan 430070, Hubei, China. ${ }^{2}$ Key Laboratory of Plant Germplasm Enhancement and Specialty Agriculture, Wuhan Botanical Garden, Chinese Academy of Sciences, Wuhan 430074, Hubei, China. ${ }^{3}$ Institute of Economic Crops, Xinjiang Academy of Agricultural Sciences, Urumqi 830091, Xinjiang, China.

\section{Received: 13 November 2020 Accepted: 17 March 2021}

\section{Published online: 25 April 2021}

\section{References}

Al Murad M, Khan AL, Muneer S. Silicon in horticultural crops: cross-talk, signaling, and tolerance mechanism under salinity stress. Plants. 2020;9(4):460. https:// doi.org/10.3390/plants9040460.

Chen J, Liu SS, Kohler A, et al. iTRAQ and RNA-Seq analyses provide new insights into regulation mechanism of symbiotic germination of Dendrobium officinale seeds (Orchidaceae). J Proteome Res. 2017;16(6):2174-87. https:// doi.org/10.1021/acs.jproteome.6b00999.

Chen L, Sun H, Wang FJ, et al. Genome-wide identification of MAPK cascade genes reveals the GhMAP3K14-GhMKK11-GhMPK31 pathway is involved in the drought response in cotton. Plant Mol Biol. 2020;103(1-2):211-23. https:// doi.org/10.1007/s11103-020-00986-0.
Chen QZ, Guo WS, Feng LZ, et al. Transcriptome and proteome analysis of Eucalyptus infected with Calonectria pseudoreteaudii. J Proteome. 2015;115(6): 117-31. https://doi.org/10.1016/j.jprot.2014.12.008.

Gao YF, Liu JK, Yang FM, et al. The WRKY transcription factor WRKY8 promotes resistance to pathogen infection and mediates drought and salt stress tolerance in Solanum lycopersicum. Physiol Plant. 2020;168(1):98-117. https:// doi.org/10.1111/ppl.12978.

Gong WF, Xu FF, Sun JL, et al. iTRAQ-based comparative proteomic analysis of seedling leaves of two upland cotton genotypes differing in salt tolerance. Front Plant Sci. 2017:8:2113. https://doi.org/10.3389/fpls.2017.02113.

Goossens J, Fernandez-Calvo P, Schweizer F, et al. Jasmonates: signal transduction components and their roles in environmental stress responses. Plant Mol Biol. 2016;91(6):673-89. https://doi.org/10.1007/s11103-016-0480-9.

Guo JY, Shi GY, Guo XY, et al. Transcriptome analysis reveals that distinct metabolic pathways operate in salt-tolerant and salt-sensitive upland cotton varieties subjected to salinity stress. Plant Sci. 2015;238:33-45. https://doi. org/10.1016/j.plantsci.2015.05.013.

He X, Zhu LF, Xu L, et al. GhATAF1, a NAC transcription factor, confers abiotic and biotic stress responses by regulating phytohormonal signaling networks. Plant Cell Rep. 2016;35(10):2167-79. https://doi.org/10.1007/s00299-016-2027-6.

Jia FJ, Qi SD, Li H, et al. Overexpression of Late Embryogenesis Abundant 14 enhances Arabidopsis salt stress tolerance. Biochem Biophys Res Commun. 2014;454(4):505-11. https://doi.org/10.1016/j.bbrc.2014.10.136.

Kang DJ, Seo YJ, Lee JD, et al. Jasmonic acid differentially affects growth, ion uptake and abscisic acid concentration in salt-tolerant and salt-sensitive rice cultivars. J Agron Crop Sci. 2005;191(4):273-82. https://doi.org/10.1111/j.1439037X.2005.00153.X.

Krishnamurthy P, Vishal B, Ho WJ, et al. Regulation of a cytochrome P450 gene CYP94B1 by WRKY33 transcription factor controls apoplastic barrier formation in roots to confer salt tolerance. Plant Physiol. 2020;184(4):2199-215. https:// doi.org/10.1104/pp.20.01054.

Li W, Zhao FA, Fang WP, et al. Identification of early salt stress responsive proteins in seedling roots of upland cotton (Gossypium hirsutum L.) employing iTRAQ-based proteomic technique. Front Plant Sci. 2015;6:732. https://doi.org/10.3389/fpls.2015.00732.

Li Z, Li L, Zhou KH, et al. GhWRKY6 acts as a negative regulator in both transgenic Arabidopsis and cotton during drought and salt stress. Front Genet. 2019;10:392. https://doi.org/10.3389/fgene.2019.00392.

Liu LL, Ren HM, Chen LQ, et al. A protein kinase, calcineurin B-like proteininteracting protein Kinase9, interacts with calcium sensor calcineurin B-like protein3 and regulates potassium homeostasis under low-potassium stress in Arabidopsis. Plant Physiol. 2013;161(1):266-77. https://doi.org/10.1104/ pp.112.206896.

Liu SH, Zhang PY, Li CC, Xia G. The moss jasmonate ZIM-domain protein PnJAZ1 confers salinity tolerance via crosstalk with the abscisic acid signalling pathway. Plant Sci. 2019;280:1-11. https://doi.org/10.1016/j.plantsci.2018.11.004.

Liu YD, Yin ZJ, Yu JW, et al. Improved salt tolerance and delayed leaf senescence in transgenic cotton expressing the Agrobacterium IPT gene. Biol Plant. 2012; 56(2):237-46. https://doi.org/10.1007/s10535-012-0082-6.

Mahajan S, Tuteja N. Cold, salinity and drought stresses: an overview. Arch Biochem Biophys. 2005;444(2):139-58. https://doi.org/10.1016/j.abb.2005.10.018.

Meloni DA, Oliva MA, Martinez CA, et al. Photosynthesis and activity of superoxide dismutase, peroxidase and glutathione reductase in cotton under salt stress. Environ Exp Bot. 2003;49(1):69-76. https//doi.org/10.1016/s0098-8472(02)00058-8.

Muchate NS, Nikalje GC, Rajurkar NS, et al. Plant salt stress: adaptive responses, tolerance mechanism and bioengineering for salt tolerance. Bot Rev. 2016; 82(4):371-406. https://doi.org/10.1007/s12229-016-9173-y.

Munns R, Tester M. Mechanisms of salinity tolerance. Annu Rev Plant Biol. 2008; 59(1):651-81. https://doi.org/10.1146/annurev.arplant.59.032607.092911.

Pandey GK, Kanwar P, Singh A, et al. Calcineurin B-like protein-interacting protein kinase CIPK21 regulates osmotic and salt stress responses in Arabidopsis. Plant Physiol. 2015;169(1):780-92. https://doi.org/10.1 104/pp.15.00623.

Park HJ, Kim WY, Yun DJ. A new insight of salt stress signaling in plant. Mol Cells. 2016;39(6):447-59. https://doi.org/10.14348/molcells.2016.0083.

Peng Z, He SP, Gong WF, et al. Comprehensive analysis of differentially expressed genes and transcriptional regulation induced by salt stress in two contrasting cotton genotypes. BMC Genomics. 2014;15(1):760. https://doi.org/10.1186/14 71-2164-15-760.

Peng Z, He SP, Gong WF, et al. Integration of proteomic and transcriptomic profiles reveals multiple levels of genetic regulation of salt tolerance in cotton. BMC Plant Biol. 2018;18(1):128. https://doi.org/10.1186/s12870-018-1350-1. 
Qiu ZB, Guo JL, Zhu AJ, et al. Exogenous jasmonic acid can enhance tolerance of wheat seedlings to salt stress. Ecotoxicol Environ Saf. 2014;104:202-8. https:// doi.org/10.1016/j.ecoenv.2014.03.014.

Ryu H, Cho YG. Plant hormones in salt stress tolerance. J Plant Biol. 2015;58(3): 147-55. https://doi.org/10.1007/s12374-015-0103-Z.

Shan Y, Zhao P, Liu Z, et al. An isopentyl transferase gene driven by the senescence-inducible SAG12 promoter improves salinity stress tolerance in cotton. J Cotton Res. 2019;2:15. https://doi.org/10.1186/s42397-019-0032-3.

Sikder RK, Wang XR, Jin DS, et al. Screening and evaluation of reliable traits of upland cotton (Gossypium hirsutum L.) genotypes for salt tolerance at the seedling growth stage. J Cotton Res. 2020;3:11. https://doi.org/10.1186/s423 97-020-00049-1.

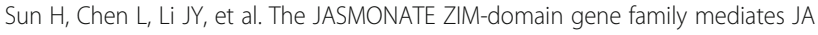
signaling and stress response in cotton. Plant Cell Physiol. 2017;58(12):213954. https://doi.org/10.1093/pcp/pcx148.

Sun $\mathrm{H}, \mathrm{Hu} \mathrm{ML}$, Li JY, et al. Comprehensive analysis of NAC transcription factors uncovers their roles during fiber development and stress response in cotton. BMC Plant Biol. 2018;18(1):150. https://doi.org/10.11 86/s12870-018-1367-5.

Tang RJ, Zhao FG, Garcia VJ, et al. Tonoplast CBL-CIPK calcium signaling network regulates magnesium homeostasis in Arabidopsis. Proc Natl Acad Sci U S A. 2015;112(10):3134-9. https://doi.org/10.1073/pnas.1420944112.

Teige M, Scheikl E, Eulgem T, et al. The MKK2 pathway mediates cold and salt stress signaling in Arabidopsis. Mol Cell. 2004;15(1):141-52. https://doi.org/1 0.1016/j.molcel.2004.06.023.

Trevisan S, Manoli A, Ravazzolo L, et al. Nitrate sensing by the maize root apex transition zone: a merged transcriptomic and proteomic survey. J Exp Bot. 2015;66(13):3699-715. https://doi.org/10.1093/jxb/erv165.

Vishwakarma K, Upadhyay N, Kumar N, et al. Abscisic acid signaling and abiotic stress tolerance in plants: a review on current knowledge and future prospects. Front Plant Sci. 2017:8:161. https://doi.org/10.3389/fpls.2 017.00161.

Wang $\mathrm{SH}$, You ZY, Ye LP, et al. Quantitative proteomic and transcriptomic analyses of molecular mechanisms associated with low silk production in silkworm Bombyx mori. J Proteome Res. 2014;13(2):735-51. https://doi.org/1 0.1021/pr4008333.

Wang XC, Li Q, Jin X, et al. Quantitative proteomics and transcriptomics reveal key metabolic processes associated with cotton fiber initiation. J Proteome. 2015;114:16-27. https://doi.org/10.1016/j.jprot.2014.10.022.

Wang Z, Hong YC, Zhu GT, et al. Loss of salt tolerance during tomato domestication conferred by variation in a $\mathrm{Na}^{+} / \mathrm{K}^{+}$transporter. EMBO J. 2020; 39(10):e103256. https://doi.org/10.15252/embj.2019103256.

Wen B, Zhou R, Feng Q, et al. IQuant: an automated pipeline for quantitative proteomics based upon isobaric tags. Proteomics. 2014;14(20):2280-5. https://doi.org/10.1002/pmic.201300361.

Xie C, Mao XZ, Huang JJ, et al. KOBAS 2.0: a web server for annotation and identification of enriched pathways and diseases. Nucleic Acids Res. 2011;39: W316-22. https://doi.org/10.1093/nar/gkr483.

Xie Z, Li D, Wang L, et al. Role of the stomatal development regulators FLP/ MYB88 in abiotic stress responses. Plant J. 2010;64(5):731-9. https://doi.org/1 0.1111/j.1365-313X.2010.04364.X.

Xie ZX, Duan LS, Li ZH, et al. Dose-dependent effects of Coronatine on cotton seedling growth under salt stress. J Plant Growth Regul. 2015;34(3):651-64. https://doi.org/10.1007/s00344-015-9501-1.

Yang X, Xu Y, Yang F, et al. Genome-wide identification of OSCA gene family and their potential function in the regulation of dehydration and salt stress in Gossypium hirsutum. J Cotton Res. 2019;2:11. https://doi.org/10.1186/s42397019-0028-z.

Yang YQ, Guo Y. Elucidating the molecular mechanisms mediating plant salt-stress responses. New Phytol. 2018a;217(2):523-39. https://doi.org/10.1111/nph.14920.

Yang YQ, Guo Y. Unraveling salt stress signaling in plants. J Integr Plant Biol. 2018b;60(9):796-804. https://doi.org/10.1111/jipb.12689.

Zelm EV, Zhang YX, Testerink C. Salt tolerance mechanisms of plants. Annu Rev Plant Biol. 2020;71(1):403-33. https://doi.org/10.1146/annurev-arplant-05071 8100005

Zhao Y, Yang Z, Ding Y, et al. Over-expression of an R2R3 MYB Gene, GhMYB73, increases tolerance to salt stress in transgenic Arabidopsis. Plant Sci. 2019; 286:28-36. https://doi.org/10.1016/j.plantsci.2019.05.021

Zhang M, Liang XY, Wang LM, et al. A HAK family $\mathrm{Na}^{+}$transporter confers natural variation of salt tolerance in maize. Nat Plants. 2019;5(12):1297-308. https:// doi.org/10.1038/s41477-019-0565-y.
Zhang TZ, Hu Y, Jiang WK, et al. Sequencing of allotetraploid cotton (Gossypium hirsutum L. acc. TM-1) provides a resource for fiber improvement. Nat Biotechnol. 2015;33(5):531-7. https://doi.org/10.1038/nbt.3207.

Zorb C, Geilfus CM, Dietz KJ. Salinity and crop yield. Plant Biol. 2019;1(S1):31-8. https://doi.org/10.1111/plb.12884.

\section{Ready to submit your research? Choose BMC and benefit from:}

- fast, convenient online submission

- thorough peer review by experienced researchers in your field

- rapid publication on acceptance

- support for research data, including large and complex data types

- gold Open Access which fosters wider collaboration and increased citations

- maximum visibility for your research: over $100 \mathrm{M}$ website views per year

At BMC, research is always in progress.

Learn more biomedcentral.com/submissions 